\title{
Contrast-enhanced sonography in pediatrics
}

\author{
M. Beth McCarville
}

Received: 17 December 2010/Revised: 13 January 2011 /Accepted: 24 January 2011

(C) Springer-Verlag 2011

\begin{abstract}
Microbubble US contrast agents are composed of an outer shell of protein, phospholipid or polymer that encase air or perfluorocarbon gas. These contrast agents have been widely used in adult cardiology patients to improve endocardial border delineation and have been proved safe and well tolerated in this patient population. There is also a growing body of literature elucidating the value of contrast-enhanced sonography to distinguish benign from malignant liver lesions in adults and to characterize non-hepatic adult malignancies. Because these agents have not been approved for pediatric use in many countries, less is known of the value of contrast-enhanced sonography in children. In this review I will discuss several proven and potential pediatric applications of contrast-enhanced sonography.
\end{abstract}

Keywords Microbubble contrast agent · Sonography Pediatrics

\section{Introduction}

Ultrasound (US) is an ideal imaging modality for the pediatric population because, relative to adults, the smaller size of patients lends itself to better image resolution. Perhaps more important, it lacks ionizing radiation and has the benefit of not requiring sedation. It would seem

Disclaimer The supplement this article is part of is not sponsored by the industry. Dr. McCarville has no financial interests, to disclose.

She receives product support from GE Healthcare for off-label use of Optison ${ }^{\mathrm{TM}}$.

\section{B. McCarville $(\square)$}

Department of Radiological Sciences MS 210,

St. Jude Children's Research Hospital,

262 Danny Thomas Place,

Memphis, TN 38105, USA

e-mail: beth.mccarville@stjude.org appropriate then, to expand the role of pediatric US with the introduction of microbubble US contrast agents. These contrast agents are composed of an outer shell of protein, phospholipid or polymer that encases air or perfluorocarbon gas. Because they approximate the size of a red blood cell, they remain in the vascular space [1].

These agents have been proved safe and well tolerated in adults [2-5]. In the United States they are approved by the Food and Drug Administration (FDA) for use in adult cardiology patients because they substantially improve endocardial border delineation when baseline, noncontrast-enhanced echocardiography is nondiagnostic. In October of 2007 the FDA issued a "black-box" warning for these agents, indicating several new disease state contraindications and a mandated 30-min post-procedural monitoring period. The warning was based on postmarketing reports of four deaths and approximately 190 serious cardiopulmonary reactions that were temporally, but not clearly causally, related to US contrast agent administration [2-5]. In reaction to the FDA warning numerous cardiology groups performed large retrospective comparative analyses of adverse events in patient cohorts undergoing contrastenhanced echocardiography versus non-contrast-enhanced echocardiography. These studies uniformly found no difference in post-procedural serious adverse events between the cohorts. These findings, coupled with other efforts led by the FDA, led to a revision of the FDA recommendations in May of 2008, with removal of the specific disease state contraindications and lifting of the 30-min post-procedural monitoring mandate except in special circumstances [2-5].

In addition to the proven value in echocardiography there is a growing body of literature elucidating the value of contrast-enhanced sonography (CEUS) in distinguishing benign from malignant liver tumors and in the evaluation of non-hepatic malignancies in adults [6-9]. More recently, reports indicate their value for quantifying solid tumor 
response to anti-angiogenic agents [10]. Unlike the extensive experience of CEUS in adults, the role of US contrast agents in pediatric sonography, other than for vesicoureteral reflux and visualization of the cardiac chambers, has not been well defined. A limitation of investigating the role of CEUS in the pediatric oncological population is the relative rarity of pediatric malignancies compared to adults. Such studies, if undertaken at a single institution, would require an excessively long period of time to accrue an adequate number of subjects. In the United States this problem is compounded by the lack of FDA approval for the use of US contrast agents in pediatric patients. Large, cooperative, multi-institutional trials will be needed to fully investigate the value of CEUS in pediatrics and to obtain FDA approval for pediatric indications. Despite these drawbacks, because of the proven value of CEUS in adults and the many attributes of sonography in the pediatric population, CEUS remains an appealing modality for the assessment of a variety of pediatric conditions. This review will focus on several potential and accepted applications of CEUS in pediatric patients.

\section{Vesicoureteral reflux}

Contrast-enhanced voiding urosonography (VUS) entails the intravesical instillation of an US contrast agent to detect vesicoureteral reflux (VUR) (Fig. 1). This technique has been accepted in several European countries where US contrast agents are approved for this application. There have been no serious adverse events associated with VUS [11].

Most investigators have compared VUS to voiding cystourethrography (VCUG) as the reference standard. Using a first-generation contrast agent and fundamental imaging the diagnostic accuracy of VUS has ranged from $78 \%$ to $96 \%$, with most studies showing accuracy of $90 \%$ or above. The overall agreement between the modalities is approximately $91 \%$. Seventy percent of reflux episodes missed on VCUG but detected with VUS were grades IIIV, while $68 \%$ of episodes detected by VCUG but not VUS were grade I. The rate of detection of VCUG-missed refluxes, using VUS, increased with evolution of US technology. Studies performed with fundamental imaging detected $18 \%$ of VCUG-missed vesicoureteral reflux, color Doppler detected $21 \%$ and harmonic imaging, 28\%. Several factors might explain these findings. Because reflux can be intermittent, the use of intermittent fluoroscopy might miss episodes, whereas VUS is used continuously throughout bladder filling and voiding and is thus more likely to detect intermittent reflux. US might be more sensitive to the detection of very small amounts of refluxed microbubbles compared to small amounts of refluxed iodinated contrast
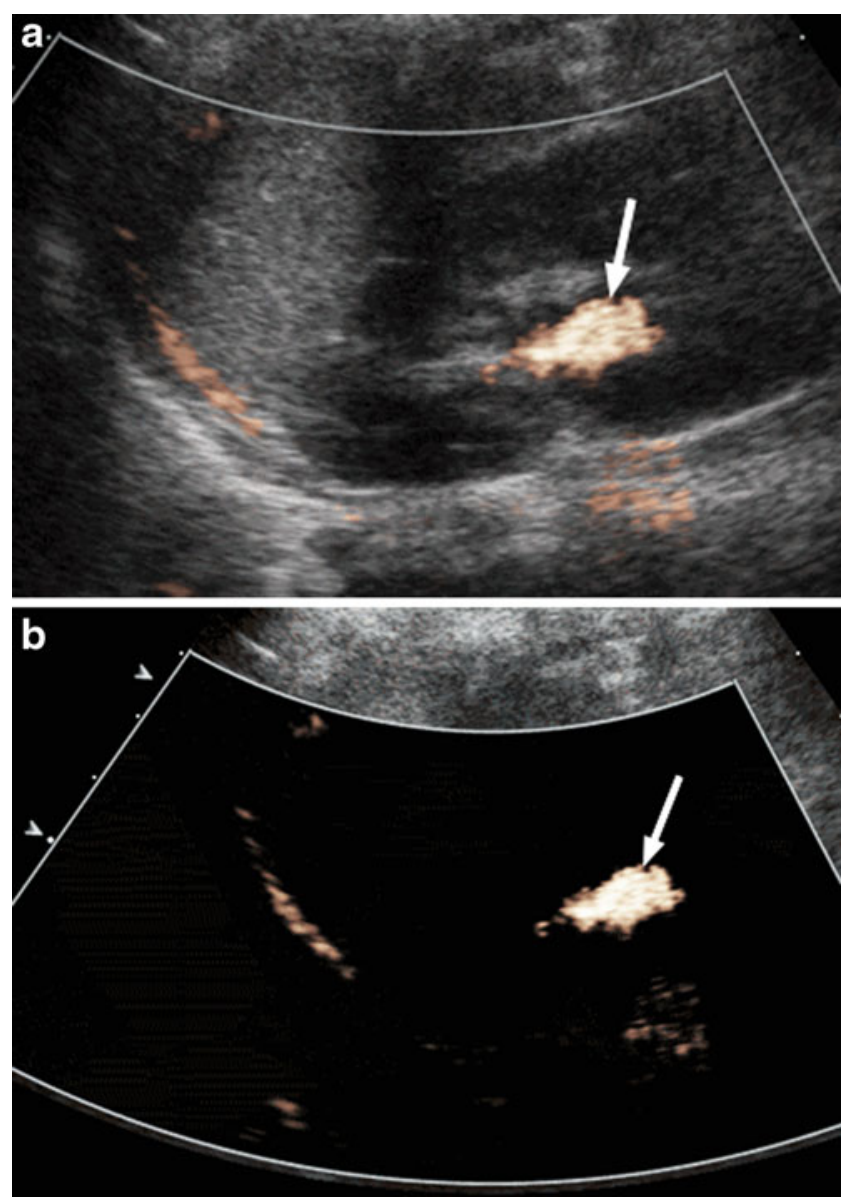

Fig. 1 VUS. a Using contrast-specific software the US contrast agent is readily visible in the renal collecting system (arrow, orange material) of this patient with grade II vesicoureteral reflux. b The software can subtract out background tissue so that the contrast agent alone is visualized (arrow, orange material). This technique improves the sensitivity for detection of contrast agent (images courtesy of Dr. Kassa Darge)

material that can be difficult to detect fluoroscopically. On the other hand, several limitations of VUS can compromise detection of VUR. One cannot image the entire urinary tract simultaneously but must focus on specific areas consecutively. Also, the contrast material, if not administered appropriately, can cause distal acoustic shadowing that can obscure the distal ureters [12]. Notably, evaluation of the urethra using VUS has been shown to be feasible and accurate compared to VCUG [13].

\section{Hemangioendothelioma vs. hepatoblastoma}

In young children the differential diagnosis of liver lesions includes hemangioendothelioma and hepatoblastoma. Hemangioendothelioma is a benign neoplasm composed of a network of capillary-size endothelium-lined vessels. Patients usually present during infancy with abdominal 
distension and hepatomegaly. Complications of hemangioendothelioma include congestive heart failure caused by intra-tumoral arterial shunting, anemia, thrombocytopenia, jaundice and bleeding [14]. Hepatoblastoma is a malignant liver tumor that occurs at a median age of 18 months. These children also often present with an abdominal mass and commonly are thrombocytopenic [15]. The serum alphafetoprotein can be elevated in both conditions [14].

In adults the addition of microbubble contrast agents has greatly improved the ability to differentiate malignant from benign liver lesions sonographically. Contrast-enhanced sonography is performed in phases similar to three-phase $\mathrm{CT}$ and MRI. The arterial phase occurs between visualization of contrast material in the hepatic arteries and contrast opacification of the main portal vein, generally $2-10 \mathrm{~s}$ after an intravenous bolus injection. In the arterial phase, the presence of lesional vessels as well as their distribution and morphology can be useful in the diagnosis of liver masses. Hypervascular masses such as hepatocellular carcinoma and focal nodular hyperplasia show linear lesion vascularity. A stellate vascular pattern is often seen in FNH, whereas HCC demonstrates dysmorphic vascularity. Hemangiomas infrequently demonstrate linear vascularity and instead show peripheral puddling of contrast material. Compared to normal adjacent liver, hypervascular lesions such as FNH and HCC appear hyperechoic in the arterial phase, whereas hypovascular lesions such as metastases appear hypoechoic $[1,16]$.

The portal-venous phase occurs $45-75 \mathrm{~s}$ after the arrival of the contrast agent into the field of view. Because the liver is supplied predominantly by the portal vein, the liver shows progressively increased enhancement throughout the arterial phase into the portal venous phase. Malignant lesions, whether metastatic or primary, derive their vascular supply from the hepatic artery and, therefore, appear hypoechoic compared to the normal liver during the portal venous phase. Additionally, malignant lesions usually show washout of contrast material during and after the portal venous phase. Sustained enhancement or increased enhancement beyond the portal venous phase has been shown to be a feature of benign lesions such as hemangioma. Hemangiomas often demonstrate progressive centripedal enhancement with complete filling-in of the lesion after the portal-venous phase, unless there is central scarring or necrosis [1, 16].

In one small study of seven infants with hemangioendotheliomas, Feng and colleagues [14] showed that on threephase CT and MRI these lesions demonstrate enhancement patterns similar to those described for adult cavernous hemangiomas. Specifically, in the arterial phase peripheral enhancement was seen in $52 \%$, homogeneous enhancement in $48 \%$, fibrillary enhancement in $33 \%$ and nodular enhancement in $29 \%$. All lesions $<1 \mathrm{~cm}$ in diameter demonstrated homogeneous enhancement in the arterial phase whereas larger lesions showed a variety of enhance- ment patterns. All tumors showed progressively increased enhancement in the portal venous phase. In the delayed phase the majority of lesions became homogeneously enhanced although larger lesions lacked complete enhancement, probably because of central necrosis or fibrosis [14].

Although there have been no reports of the three-phase enhancement pattern of hepatoblastoma, the tumor receives its vascular supply from the hepatic artery and could be expected to have enhancement features similar to those of adult HCC. Given our current understanding of contrastenhanced US in adult liver lesions it seems reasonable to apply a similar approach to pediatric liver masses. In particular, CEUS might prove useful in distinguishing hepatoblastoma from hemangioendothelioma. Because these lesions can have overlapping clinical and imaging features, CEUS could minimize exposure of these particularly vulnerable young patients to the harmful effects of radiation.

\section{Brain injury in premature infants}

Preterm infants have fragile, immature capillaries in the basal ganglia, and disturbances in the cerebral hemodynamics, particularly hyperperfusion secondary to increased cerebral blood volume (CBV) and cerebral blood flow (CBF), or increased venous pressure can lead to periventricular or intraventricular hemorrhage. Conversely, hypoperfusion secondary to decreased cerebral blood flow leads to hypoxic-ischemic injury and periventricular leukomalacia. There are currently no reliable, non-invasive methods to continuously monitor $\mathrm{CBF}$ and $\mathrm{CBV}$ in premature infants. Doppler US evaluation is limited to a single, large cerebral artery and cannot provide information regarding the brain microcirculation [17].

During the last decade considerable progress has been made in the application of CEUS in adults with suspected acute stroke to qualitatively, and more recently quantitatively, assess cerebral microperfusion. In one study by Seidel and colleagues [18], 24 adults with acute middle cerebral artery (MCA) infarctions underwent contrastenhanced transcranial US studies that were compared to non-contrast-enhanced head CTs performed on the same day and within $12 \mathrm{~h}$ of symptom onset (baseline). Follow-up head CT was performed within $34 \mathrm{~h}$ and follow-up CEUS within $78 \mathrm{~h}$ of initial presentation. The baseline CEUS qualitatively demonstrated areas of hypoperfusion, relative to normal brain, in $79 \%$ of patients in areas that were demarcated on the follow-up head CTs. Analysis of brain regions-of-interest and time-intensity curves showed that this method had $86 \%$ sensitivity and $96 \%$ specificity for localizing areas of infarction [18]. More recently, these investigators reported their experience with software that is faster and 
more robust and allows quantification of US contrast agent kinetics. Their newer method showed $100 \%$ sensitivity and $100 \%$ specificity in the detection of ischemic brain tissue in 26 adults $[18,19]$.

Although MRI offers methods of assessing cerebral perfusion, it is difficult and often impractical to transport a severely ill premature infant to the radiology department for this lengthy imaging examination. In contrast, CEUS could easily be performed at the child's bedside. A limitation of transcranial US in adults is the acoustic impediment caused by the skull, which hinders visualization of cerebral anatomy and causes streak artifact. In infants the open fontanelles offer excellent acoustic windows for transcranial sonography. These attributes make transcranial CEUS even more appealing for use in infants than adults. Based on the success of transcranial CEUS in adults with cerebral vascular insult it seems feasible that both qualitative and quantitative assessment of cerebral contrast agent flow in infants with suspected deregulated cerebral perfusion could identify sites of normal, hyper- or hypoperfused brain before the sequelae become evident on conventional sonography.

\section{Assessing tumor response to anti-angiogenic agents}

The successful introduction of antiangiogenic therapies into cancer clinical trials will require reliable, noninvasive methods of assessing tumor angiogenesis and its alteration or inhibition in vivo [20]. Imaging modalities showing promise for assessing tumor blood flow include perfusion $\mathrm{CT}$, dynamic contrast-enhanced MRI, and quantitative CEUS. Because perfusion CT exposes the patient to a substantial dose of ionizing radiation, it is not an attractive option for pediatric patients. Dynamic contrast-enhanced MRI often requires sedation of young patients and can be time-consuming and costly.

Because microbubble US contrast agents remain within the vascular space they allow a surrogate method of assessing blood flow. These contrast agents are highly reflective on US imaging and offer an approach to enhance visualization of tumor neovasculature in small volumes of tissue and to monitor the early effects of antiangiogenic therapy on small tumor neovessels, whose diameter is typically less than $50 \mu \mathrm{m}$ [21].

We have shown that CEUS is a reliable method for assessing tumor blood flow in murine pediatric tumor models treated with various antiangiogenic agents [22-24]. Most recently, we investigated temporal vascular changes induced by the anti-angiogenic agent bevacizumab in a murine neuroblastoma model by evaluating tumor perfusion at $24 \mathrm{~h}, 3$ days, and 7 days after the administration of one dose of this agent. The measured intratumoral change in signal intensity from baseline to peak $(\Delta \mathrm{SI})$ was significantly increased at $24 \mathrm{~h}$ and at day 3 , in treated mice versus size-matched controls. At Day 7 , the $\Delta$ SI was almost equivalent for treated and control tumors. These findings were confirmed by intravital microscopy and immunohistochemistry, which showed vessel maturation and normalization of vascular morphology at days 1 and 3 with vascular pruning (decrease in number of vessels) at day 7 . We concluded that bevacizumab transiently improved tumor vascularity, with a subsequent increase in tumor blood flow, early after its administration but eventually reduces tumor perfusion. Such information could be important in scheduling conventional chemotherapy to optimize intratumoral drug delivery [25].

Contrast-enhanced US is emerging as a valuable and reliable method of assessing antiangiogenic therapies in adult clinical trials [26-28]. This approach allows for an objective, quantifiable method of assessing tumor blood flow. Changes in tumor perfusion detected by CEUS might ultimately predict tumor response earlier than conventional modalities using RECIST (response evaluation criteria in solid tumors), which relies on tumor shrinkage [28]. A limitation of CEUS for monitoring solid tumor response will be those primary or metastatic sites of disease that are not amenable to sonographic visualization. Despite this and because of the many attributes of sonography in the pediatric population already described, this is an area of research that should be pursued.

\section{Conclusion}

Contrast-enhanced US requires further investigation in pediatric trials. I have presented only several potential applications of CEUS that could have important clinical implications. Given the safety record of US contrast agents and the need to apply the ALARA principle, pediatric radiologists have a unique opportunity to maximize the potential of CEUS. This would necessitate multi-institutional trials and collaboration with industry and regulatory agencies.

\section{References}

1. Brannigan M, Burns PN, Wilson SR (2004) Blood flow patterns in focal liver lesions at microbubble-enhanced US. Radiographics 24:921-935

2. Piscaglia F, Bolondi L (2006) The safety of Sonovue in abdominal applications: retrospective analysis of 23, 188 investigations. Ultrasound Med Biol 32:1369-1375

3. Abdelmoneim SS, Bernier M, Scott CG et al (2009) Safety of contrast agent use during stress echocardiography: a 4-year experience from a single-center cohort study of 26, 774 patients. JACC Cardiovasc Imaging 2:1048-1056 
4. Main ML, Goldman JH, Grayburn PA (2009) Ultrasound contrast agents: balancing safety versus efficacy. Expert Opin Drug Saf 8:49-56

5. Wei K (2010) Contrast echocardiography: what have we learned from the new guidelines? Curr Cardiol Rep 12:237-242

6. Moritz JD, Ludwig A, Oestmann JW (2000) Contrast-enhanced color Doppler sonography for evaluation of enlarged cervical lymph nodes in head and neck tumors. AJR 174:1279-1284

7. Nicolau C, Bunesch L, Peri L et al (2010) Accuracy of contrastenhanced ultrasound in the detection of bladder cancer. $\mathrm{Br} \mathrm{J}$ Radiol [Epub ahead of print] 1 Dec

8. Sano F, Terao H, Kawahara T et al (2010) Contrast-enhanced ultrasonography of the prostate: various imaging findings that indicate prostate cancer. BJU Int [Epub ahead of print] 29 Oct

9. Seicean A, Badea R, Stan-Iuga R et al (2010) Quantitative contrastenhanced harmonic endoscopic ultrasonography for the discrimination of solid pancreatic masses. Ultraschall Med 31:571-576

10. Weskott HP (2008) Emerging roles for contrast-enhanced ultrasound. Clin Hemorheol Microcirc 40:51-71

11. Darge K (2010) Voiding urosonography with US contrast agent for the diagnosis of vesicoureteric reflux in children: an update. Pediatr Radiol 40:956-962

12. Darge K (2008) Voiding urosonography with US contrast agents for the diagnosis of vesicoureteric reflux in children. II. Comparison with radiological examinations. Pediatr Radiol 38:54-63

13. Berrocal T, Gaya F, Arjonilla A (2005) Vesicoureteral reflux: can the urethra be adequately assessed by using contrast-enhanced voiding US of the bladder? Radiology 234:235-241

14. Feng ST, Chan T, Ching AS et al (2010) CT and MR imaging characteristics of infantile hepatic hemangioendothelioma. Eur J Radiol 76:e24-e29

15. Roebuck DJ (2009) Assessment of malignant liver tumors in children. Cancer Imaging 9 Spec No A:S98-S103

16. Wilson SR, Burns PN (2001) Liver mass evaluation with ultrasound: the impact of microbubble contrast agents and pulse inversion imaging. Semin Liver Dis 21:147-159

17. Liem KD, Greisen G (2010) Monitoring of cerebral haemodynamics in newborn infants. Early Hum Dev 86:155-158
18. Seidel G, Albers T, Meyer K et al (2003) Perfusion harmonic imaging in acute middle cerebral artery infarction. Ultrasound Med Biol 29:1245-1251

19. Maciak A, Seidel G, Meyer-Wiethe K et al (2008) Automatic detection of perfusion deficits with bolus harmonic imaging. Ultraschall Med 29:618-626

20. Anderson H, Price P, Blomley $M$ et al (2001) Measuring changes in human tumour vasculature in response to therapy using functional imaging techniques. Br J Cancer 85:1085-1093

21. Ferrara KW, Merritt CR, Burns PN et al (2000) Evaluation of tumor angiogenesis with US: imaging, Doppler, and contrast agents. Acad Radiol 7:824-839

22. McCarville MB, Streck CJ, Dickson PV et al (2006) Angiogenesis inhibitors in a murine neuroblastoma model: quantitative assessment of intratumoral blood flow with contrast-enhanced grayscale US. Radiology 240:73-81

23. Dickson PV, Hamner JB, Sims TL et al (2007) Bevacizumabinduced transient remodeling of the vasculature in neuroblastoma xenografts results in improved delivery and efficacy of systemically administered chemotherapy. Clin Cancer Res 13:3942-3950

24. Dickson PV, Hamner JB, Streck CJ et al (2007) Continuous delivery of IFN-beta promotes sustained maturation of intratumoral vasculature. Mol Cancer Res 5:531-542

25. Mandai M, Koda M, Matono T et al (2010) Assessment of hepatocellular carcinoma by contrast-enhanced ultrasound with perfluorobutane microbubbles: comparison with dynamic CT. Br J Radiol [Epub ahead of print] 19 Oct

26. Lassau N, Koscielny S, Chami L et al (2010) Advanced hepatocellular carcinoma: early evaluation of response to bevacizumab therapy at dynamic contrast-enhanced US with quantification - preliminary results. Radiology 258:291-300

27. Lassau N, Chebil M, Chami L et al (2010) Dynamic contrastenhanced ultrasonography (DCE-US): a new tool for the early evaluation of antiangiogenic treatment. Target Oncol 5:53-58

28. Eisenhauer EA, Therasse P, Bogaerts J et al (2009) New response evaluation criteria in solid tumours: revised RECIST guideline (version 1.1). Eur J Cancer 45:228-247 